\title{
Erratum to: High yield production of ultrathin fibroid semiconducting nanowire of $\mathrm{Ta}_{2} \mathrm{Pd}_{3} \mathrm{Se}_{8}$
}

Xue Liu ${ }^{1,2}$, Sheng Liu' ${ }^{2}$, Liubov Yu. Antipina ${ }^{3,5}$, Yibo Zhu ${ }^{7}$, Jinliang Ning ${ }^{1}$, Jinyu Liu ${ }^{1}$, Chunlei Yue ${ }^{1}$, Abin Joshy ${ }^{1}$, Yu Zhu' ${ }^{4}$, Jianwei Sun ${ }^{1}$, Ana M. Sanchez ${ }^{8}$, Pavel B. Sorokin ${ }^{3,6}$, Zhiqiang Mao ${ }^{1,9}$, Qihua Xiong ${ }^{2}$, and Jiang Wei ${ }^{1}(\bowtie)$

${ }^{1}$ Department of Physics and Engineering Physics, Tulane University, New Orleans, Louisiana 70118, USA

${ }^{2}$ Division of Physics and Applied Physics, Nanyang Technological University, Singapore 637371, Singapore

${ }^{3}$ National University of Science and Technology "MISiS", Moscow 119049, Russia

${ }^{4}$ Department of Polymer Science, The University of Akron, Akron, Ohio 44325, USA

${ }^{5}$ Emanuel Institute of Biochemical Physics of RAS, Moscow 119334, Russia

${ }^{6}$ Moscow Institute of Physics and Technology(State University), Dolgoprudny 141700, Russia

${ }^{7}$ Department of Mechanical Engineering, Columbia University, New York, New York 10027, USA

${ }^{8}$ Department of Physics, University of Warwick, Coventry, CV4 7AL, UK

${ }^{9}$ Department of Physics, the Pennsylvania State University, University Park, PA 16802, USA

(C) Tsinghua University Press and Springer-Verlag GmbH Germany, part of Springer Nature 2020

\section{Erratum to}

Nano Research 2020, 13(6): 1627-1635

https://doi.org/10.1007/s12274-020-2784-y

The author information and the Acknowledgement were unfortunately incorrect,

Instead of

Xue Liu ${ }^{1,2}$, Sheng Liü ${ }^{2}$, Liubov Yu. Antipina ${ }^{3,5,6}$, Yibo Zhu ${ }^{7}$, Jinliang Ning ${ }^{1}$, Jinyu Liu ${ }^{1}$, Chunlei Yue ${ }^{1}$, Abin Joshy ${ }^{1}$, Yu Zhu ${ }^{4}$, Jianwei Sun ${ }^{1}$, Ana M Sanchez ${ }^{8}$, Pavel B. Sorokin ${ }^{3,5}$, Zhiqiang Mao ${ }^{1}$, Qihua Xiong ${ }^{2}$, and Jiang Wei ${ }^{1}(\bowtie)$

${ }^{1}$ Department of Physics and Engineering Physics, Tulane University, New Orleans, Louisiana 70118, USA

${ }^{2}$ Division of Physics and Applied Physics, Nanyang Technological University, Singapore 637371, Singapore

${ }^{3}$ National University of Science and Technology "MISiS", Moscow 119049, Russia

${ }^{4}$ Department of Polymer Science, The University of Akron, Akron, Ohio 44325, USA

${ }^{5}$ Technological Institute for Superhard and Novel Carbon Materials, Moscow 108840, Russia

${ }^{6}$ Moscow Institute of Physics and Technology, Dolgoprudny 141700, Russia

${ }^{7}$ Department of Mechanical Engineering, Columbia University, New York, New York 10027, USA

${ }^{8}$ Department of Physics, University of Warwick, Coventry, CV4 7AL, UK

\section{Acknowledgements}

This work is supported by the United States Department of Energy under Grant DE-SC0014208 and by The National Science Foundation under Grant 1752997. We acknowledge the Coordinated Instrument Facility (CIF) of Tulane University for the support of various instruments. P. B. S. and L.Y. A. (theoretical calculations) were supported by the Russian Science Foundation (No. 17-72-20223). We are grateful to the supercomputer cluster provided by the Materials Modelling and Development Laboratory at NUST "MISIS" (supported via the Grant from the Ministry of Education and Science of the Russian Federation No. 14.Y26.31.0005) and to the Joint Supercomputer Center of the Russian Academy of Sciences.

It should read

Xue Liu ${ }^{1,2}$, Sheng Liü ${ }^{2}$, Liubov Yu. Antipina ${ }^{3,5}$, Yibo Zhu ${ }^{7}$, Jinliang Ning ${ }^{1}$, Jinyu Liu ${ }^{1}$, Chunlei Yue ${ }^{1}$, Abin Joshy ${ }^{1}$, Yu Zhu ${ }^{4}$, Jianwei Sun ${ }^{1}$, Ana M. Sanchez ${ }^{8}$, Pavel B. Sorokin ${ }^{3,6}$, Zhiqiang Mao ${ }^{1,9}$, Qihua Xiong ${ }^{2}$, and Jiang Wei ${ }^{1}(\bowtie \Delta)$

${ }^{1}$ Department of Physics and Engineering Physics, Tulane University, New Orleans, Louisiana 70118, USA

${ }^{2}$ Division of Physics and Applied Physics, Nanyang Technological University, Singapore 637371, Singapore 
${ }^{3}$ National University of Science and Technology "MISiS", Moscow 119049, Russia

${ }^{4}$ Department of Polymer Science, The University of Akron, Akron, Ohio 44325, USA

${ }^{5}$ Emanuel Institute of Biochemical Physics of RAS, Moscow 119334, Russia

${ }^{6}$ Moscow Institute of Physics and Technology(State University), Dolgoprudny 141700, Russia

${ }^{7}$ Department of Mechanical Engineering, Columbia University, New York, New York 10027, USA

${ }^{8}$ Department of Physics, University of Warwick, Coventry, CV4 7AL, UK

${ }^{9}$ Department of Physics, the Pennsylvania State University, University Park, PA 16802, USA

\section{Acknowledgements}

This work is supported by the United States Department of Energy under Grant DE-SC0014208 in material synthesis and partial efforts of material processing and by the National Science Foundation under Grant 1752997. We acknowledge the Coordinated Instrument Facility (CIF) of Tulane University for the support of various instruments. P. B. S. and L.Y. A. (theoretical calculations) were supported by the Russian Science Foundation (No. 17-72-20223). We are grateful to the supercomputer cluster provided by the Materials Modelling and Development Laboratory at NUST "MISIS" (supported via the Grant from the Ministry of Education and Science of the Russian Federation No. 14.Y26.31.0005) and to the Joint Supercomputer Center of the Russian Academy of Sciences.

The online version of the original article can be found at https://doi.org/10.1007/s12274-020-2784-y 